\title{
Considerazioni Intorno All'eficacia Della Sentenza
}

\section{Carlos Alberto Alvaro de Oliveira}

\author{
Ordinario di Diritto Processuale Civile \\ Università Federale del Rio Grande do Sul \\ Porto Alegre Brasile
}

1. Il problema deila efficacia della senterza resa nella procedura di cognizione è questione delicata e dibattuta tra i processualisti, sopratutto negli ultimi tempi. L'interesse siè intensificato a causa della presa di coscienza della necessità di garantire al processo una vera e propria effetività quale strumento della giurisdizione (oltre alla preocupazione legittima di costruite concetti adeguati). A volte affrontata come questione concernente il diritto processuale, a volte integrata nell'ambito del diritto sostanziale, la questione non ha avuto finora, a mio parere, soluzione soddisfacente.

Questo saggio parte dall'esame dello stato atuale della dottrina (n. 2 e 3 ), analizzata criticamente (n. 4 e 5) per arrivare, alla conclusione, con una positiva elaborazione del tema (n. 6) più adatta alle esigenze odierne.

2. Nella dottrina brasiliana emergono due teorie contrapposte.

L'opinione tradizionale fa dipendere l'efficacia della sentenza dal tipo di tutela giurisdizionale concessa dal giudice alla parte. In questo ambito, si adotta l'usuale tripartizione: sentenza di accertamento, di condanna, costitutiva.

Humberto Theodoro Jr. espone con chiarezza la prima teoria: "la più tilevante classificazione delle sentenze (sia la decisione monocratica, sia quella collegiale), è quella che prende spunto dalla natura del bene giuridico che è oggetto del giudizio, ossia, dal ripo di tutela giurisdizionale resa alla parte". Conseguentemente, Theodoro Jr. adotta la tripartizione sopra richiamata. Pet quanto tiguarda le sentenze dette mandamentale e esecutotie - che danno luogo a ordini da attuare nell'ambito stesso della procedura nella quale sono stati 
emanati - l'autore ritiene che siano catalogabili in una delle tre categorie tradizionali: "Siano quelle cosidette esecutorie che quelle mandamentale tealizzano l'essenza delle sentenze di condanna, cioè, accertano la situazione giuridica dei litiganti e ordinano una prestazione di una parte in favore dell'altra. La forma processuale attraverso la quale viene realizzata questa prestazione segna il punto di differenza. In altre parole, la differenza tisiede nella esecuzione e nel corrispettivo procedimento. Se così è, non esiste motivo per attrihuire natura diversa a queste sentenze"!

Cândido Rangel Dinamarco, nonostante alcuni aggiustamenti e modifiche da lui introdotti, aderisce sostanzialmente a questa teoria.

L'autore pone in rilievo che la classificazione ternaria delle azioni, basata sul tipo di sentenza richiesta, ha il merito di ben adattarsi al campo processuale, evitando i criteri trasmessi dalla tradizione romanistica, derivati da elementi del diritto sostanziale (inerenti al diritto soggettivo affermato dall'autore) che conducevano alle sentenze 'reali', 'personali' etc. Rileva, inoltre, che una siffatta classificazione ternaria è più coerente con la moderna impostazione del processo civile, secondo la quale le domande sono classificate come iniziative concrete volte a richiedere la tutela giurisdizionale.$^{2}$ La classificazione è ternaria: sentenze di condanna, di accertamento e costitutive. Per quanto riguarda le sentenze mandamentale, integrerebhero la classe delle sentenze di condanna: "La sentenza mandamentale è titolo per l'esecuzione, così comme lo è quella di notmale condanna. È, perciò, anch'essa una sentenza di condanna. La differenza specifica stà nel contenuto della sanzione che avviene nel momento posteriore, nel quale si intensifica il fattore commando o ordine. (...) In codeste sentenze il commando ha una rale intensità che autorizza il giudice, nel proprio processo di cognizione (senza bisogno di ricorrere al processo di esecuzione) a infliggere misure dirette a rendere all'attore vittorioso l'effettiva soddisfazione del suo diritro ${ }^{3}$. Più avanti, vienne rinforzata la stessa idea: "Non si tratta di una quarta categoria di sentenza, accanto a quelle di accertamento, di condanna o costitutiva. Secondo la sua struttura, funzione ed efficacia, le sentenze mandamentale hanno natura di condanna. (Cintra-Grinover-Dinamarco), nonostante il rafforzamento di efficaci datogli dalla legge ${ }^{\text {th4 }}$.

Dinamarco ammette, peralrro, la sottocategoria delle sentenze esecutorie, allorchè la sentenza di condanna trova esecuzione nello stesso processo nel quale è stata proferita, senza, dunque, l'istaurazione autonoma del processo esecutivo. Per il giurista, questi casi

\footnotetext{
'Humberto Theodoro Júnior, Curso de Direito Processual Civil, vol. I, 39a. ed., Rio de Janeiro, Forense, 2003, n" 497, p. 468-469, n' 499, p. 470.

${ }^{2}$ Cândido Rangel Dinamatco, Instituições de Direito Processual Civil, vol. III, 2a. ed., São Paulo, Malheiros, $2002, n^{\circ} 894$, p. $204-205$.

${ }^{3}$ Cândido Rangel Dinamarco, Instituiçōes de Direito Processual Civil, cit., n 919, p. 242-243.

* Cândido Rangel Dinamarco, Instituições de Direito Processual Civil, cit., n 919, p. 245.
} 
sono tari e eccezionali nel sistema e esistono soltanto nella misura in cui sono ammessi dal diritto positivo specificamente (il che ocorre nelle azioni di convalida di sfratto, possessorie, nelle esptopriazioni imobiliarie etc. $)^{5}$.

3. L'altra teotia prende spunto delle idee elaborate da Pontes de Miranda, posteriormente riprese e sviluppate da Ovidio A. Baptista da Silva.

Sostiene Pontes de Miranda, inanzitutto, cbe l'efficacia della sentenza è indissolubilmente legata all'azione di diritto sostanziale esercitata dall'autore. Non è per caso che, quando riferisce semplicemente azione (senza virgolette, cioè, nel senso di azione di diritto sostanziale) afferma: "L'azione, la cui nascita suppone trasgressione di una norma giuridica, costituisce un plus e si dirige ad un effetto giuridico specifico e non alla prestazione"6 . Più avanti, riafferma: "Mentte il diritto soggettivo e la pretesa tendono alla prestazione, l'azione suppone combatività e, dunque, tende all'effetto giuridico specifico e non alla prestazione" . In questa prospettiva, "Il concetto di azione, la classificazione delle azioni secondo la sua efficacia, tutto questo si riferisce al diritto materiale, poichè il fine principale del processo è la realizzazione del diritto oggettivo. Nella propria classificazione delle azioni e delle sentenze, il diritto processuale deve consentite l'efficacia delle azioni, secondo il diritto materiale. Il margine di libertà lasciatogì è ristretto, e però esiste". "

Ancora Pontes de Miranda: "Ie azioni sono o di accertamento (si tenga presente che i rapporti giuridici che hanno per contenuto diritti e pretensioni, oppure fatti dai quali essi derivano, inanzitutto esistono) o costitutive (positive o negative, cioè, che generano o modificano o estinguono rapporti giuridici), o di condanna, o mandamentale; oppure sono esecutive" " "restando superate le classificazioni fatte dai giuristi europei. Sia la classificazione binaria che quella ternaria (azione di accerramento, di condanna e costitutive) non resistono a critiche e contribuirono per creare confusioni ingenti che ancor'oggi si presentano nello spirito di alcuni giuristi i quali non si sono accorti che forza di sentenza (eficcacia preponderante) e eficcacia immediata o mediata sono cose diverse (a prescindere di quelle efficacie minori, con le quali si completa la costante di efficacia delle azioni e sentenze)". ${ }^{10}$

Secondo Pontes de Miranda, la classificazione giusta è la seguente: "a) L'azione di accertamento ha che fare con l'essere o non-essete del rapporto giutidico. b.) In generale, l'azione costitutiva si riferisce alla pretesa costitutiva, res deducta, quando si esercita una

\footnotetext{
${ }^{5}$ Cândido Rangel Dinamarco, Instituições de Direito Processual Civil, cit., n 920, p. $245-246$.

f Pontes de Miranda, Tratado das Açöes, tomo I, Säo Paulo, 1970, RT, p. 33.

'Pontes de Mitanda, Tratado, cit., p. 93.

8 Pontes de Miranda, Tratado, cit., p. 126.

- Pontes de Miranda, Tratado, cit., p. 117.

10 Pontes de Miranda, Tratado, cit, p. 118.
} 
pretesa alla tutela giuridica. Quando l'azione costitutiva si lega al diritto, immediatamente, non esiste nel piano della res in iudicium deducta pretesa costitutiva (esiste nel piano del diritto soggettivo alla tutela giuridica, che altro non è che la specializzazione, tramite l'esercizio della pretesa, alla tutela giuridica in preresa costitutiva). c.) L'azione di condanna presuppone che coloro ai quali si dirige abhiano operato contro diritto, che abbiano causato danno e meritino, perciò, di essere condannati (con-damnare). d.) L'azione mandamentale si riferisce ad atti che il giudice $o$ altra autorità ordini. Il giudice adotta la misura mandamentale perchè l'attore ha pretesa al mandamento e, esercitando la tutela giuridica, ha proposto l'azione mandamentale. e.) L'azione esecutoria è quella tramite la quale trasferisce nella sfera giuridica di una persona quello che in questa sfera doveva stare e non è invece presente." ${ }^{\text {in }}$

Si schiera al franco di Pontes de Miranda, Ovídio A. Baptista da Silva: "Riprendendo ciò che già è stato detto, dobbiamo distinguere nettamente le due forme di agire, e, perciò, le due specie di azioni: una, che si svolge nel piano del diritto sostanziale e che corrisponde all'agire contro l'obbligato al fine di realizare il diritto, prescindendo della sua collaborazione; un'altra, diretta contro lo Stato e provocata dall'interessato (attore) perchè esso (lo Stato) eserciti attività giurisdizionale (alla quale è obbligato) e renda la tispettiva tutela, adeguata alla richiesta (dell'autore). La prima specie presuppone un diritto sostanziale preesistente, del quale l'attote è titolare; l'altra, la 'azione' processuale, è pure fondata su un diritto anteriore. Anch'essa, come qualsiasi attività lecita, deve corrispondere ad un diritto esigibile (pretesa), perchè non diventi arbitrio e violenza." Più avanti, afferma: "Colui che agisce (esercita azione) sul piano processuale non può assolutamente ptescindere dalla attività dello Stato direttá alla realizazzione del diritto dell'autore alla giurisdizione. Al contrario, l'esercizio della 'azione' in questo caso, comprende sia l'agire che l'esigere l'azione dello Stato, per attuare la tutela giuridica". ${ }^{12}$

In tema di classificazione delle azioni, osserva l'autore: "Quando si classifica l'atto giurisidizionale tipico - decisioni e sentenze del 'processo di cognizione' e dell'esecuzione creditizia - si deve esaminare e ordinare il risultato di questa attività statale a seconda degli effetti che ciascuna azione produce nel mondo giuridico, e cioè, a seconda del verbo tramite il quale la sentenza riflette l'azione di diritto sostanziale corrispondente, contenuta nella domanda, dalla quale la decisione giudiziale è conseguenza necessaria."

Seguendo questo criterio, l'autore divide le sentenze, di accertamento, costitutive, di condanna, esecutorie e mandamentale, "a seconda che la pretesa di diritto sostanziale dell'autore vittorioso sia diretta a ottenere semplice dichiarazione dell'esistenza o non esistenza di un determinato rapporto giuridico; alla sua costituzione, modificazione o estinzione; alla

\footnotetext{
"Pontes de Miranda, Tratado, cit., p. 118-122.

:2 Ovidio A. Baptista da Silva, Cuxso de Processo Civil, vol. I, 5a. ed. riveduta ed atualizzata, São Paulo, RT, 2000, p. $92-93$.
} 
condanna del convenuto a compiere un'obbligazione o alla tealizzazione immediata del diritto in contestazione nel processo di cognizione, tramite un'atto di esecuzione del giudice o tramite un semplice comandamento o otdine emesso dal magistrato, come parte del contenuto della sentenza" ${ }^{i 3}$. Si veda anche: "Quando si afferma che le azioni - e le corrispettive sentenze di ptocedenza - possono essere di accertamento, costituttive o di condanna, si fa tiferimento ad azioni di dititto sostanziale che stanno alla base dell'atto introduttivo della domanda e che sono prese in considerazione all'interno del rapporto giuridico ptocessuale come semplici ipotesi di lavoro che si offrono al magistrato". ${ }^{14}$

4. È certamente insufficente il tentativo di classificare le azioni tenendo conto unicamente di fattori processuali. Sembra avet ragione Ovídio A. Baptista da Silva quando afferma: "l' 'azione' processuale, una ed astratta, non può avete contenuto di accertamento, costituttivo o di condanna, senza diventare azione della tradizione civilistica (...). Il prodigio di una qualche cosa che, non avendo sostanza - perchè è uguale a se stessa e concessa a tutti, senza discriminazione - possa essere dichiaratoria, costitutiva o di condanna è una contraddizione che non arriva a offendere la razionalità dei processualisti". ${ }^{15}$

D'altra parte, la teoria dell'azione astratta sembra non tenere nel conto dovuto il fatto che le diverse forme procedurali reagiscono sull'azione (l'agire in giudizio) e determinano poteri, facoltà e oneri diversi: un solo atto è comune a tutti i tipi di azioni (che deriva dal potere astratto di attivare la procedura), ma non si deve ignorare che gli atti posteriori possono avere dimensioni diverse, a causa dell'oggetto del processo, della forma etc. ${ }^{16}$ E questo perchè non sembra possibile respingere il legame col diritto sostanziale in virtù deila inerente strumentalità che la funzione giurisdizionale esercita in rapporto ad esse, al quale servono, azione e processo, tramite l'esercizio dei poteri, facoltà e oneri delle parti.

Il processo è sempte indissolubilmente legato al diritto sostanziale. Come osserva giustamente il Fazzalari, l'attore allega dei fatti, ma non già qualsiasi "episodio della vita" bensì dei fatti Che, rientrando nello schema di una norma, generano determinate conseguenze giuridiche. Deduce, cioè, i fatti costitutivi della situazione giuridica (sostanziale) preesistente, e, innanzitutto, la fattispecie concreta da cui sia derivata la posizione di preminenza rispetto al bene: il che val dire il diritto soggettivo (sostanziale). Si tratta, ovviamente, di realtà meramente affermata come esistente $e$ che diventa problematica dal momento in cui è oggetto di discussione, per finire come realtà accertata, nella ipotesi di sentenza di accoglimento della domanda ${ }^{17}$. Un'altro punto di contatto si avverte nella richiesta immediata che caratterizza il

1. Ovidio A. Baptista da Silva, Curso de Processo Civil, vol. 1 , cit., p. 112.

14 Ovidio A. Baptista da Silva, Curso cit, vol. 1, p. 160 .

${ }^{15}$ Ovidio A. Baptista da Silva, Jurisdição e Execução na tradiçăo romano-canônica, São Patilo, RT, 1996, p. 179.

is Elio Fazzalari, Note in tema di diritto e processo, Milano, Guffrè, 1953, p. 151, nota 129.

"Fazzalari, Note in tema di diritto e processo, cit., p. 122-123. 
tipo di tutela giurisdizionale adatta al caso (accertamento, condanna, costituzione, ordine o esecuzione), e che si presenta come strettamente legato al diritto sostanziale. La stessa lesione affermata dall'aurore - che conduce al venir meno di una situazione materiale protetta e che giustifica l'interesse nella tutela giurisdizionale richiesta - ha a che fare col diritto sostanziale.

Tutto ciò sta a dimostrare che non è possibile attenersi soltanto al diritto processuale per costruire la teoria della efficacia della sentenza.

5. D'altro verso, non è soddisfacente l'uso del poco chiaro concetto di azione di diritto sostanziale.

Si riprenda Pontes de Miranda: "L'esercizio di pretesa, azione o eccezione è esercizio del diritto che li contiene. Ad alcuni poteri corrispondo azioni dirette alla rispettiva realizzazione o 'dichiarazione'. Ecco che siamo arrivati a uno dei punti più rilevanti della Teoria Generale del Diritto: in ogni diritto, pretesa, azione od eccezione, si innesta il potere di rivelarsi, ogniqualvolta il diritto viene ostacolato o difficoltata la sua 'revelabilità'. Questo potere di rivelarsi consiste nell' l'actio e trova corrispondenza nel contenuto favorevole della sentenza, quando si esercita la pretesa alla tutela giuridicat"18. L'azione (di diritto sostanziale) è l'esaltazione del diritto o della pretensione ${ }^{19}$. "L' 'azione' è, peraltro, un'altro grado nel quale si conferisce a un soggetto, aurore, titolare dell'azione (di diritto sostanziale), il potere di esigere la verifica e l'attuazione della legge" ${ }^{\text {"20. }}$. L' 'azione' si esercita insieme con l'azione ${ }^{21}$.

Quando si afferma che "l'azione (di diritto sostanziale) è l'esaltazione del diritto o della pretesa" vengono subito alla memoria le idee di Savigny il quale considerava l'azione di diritto sostanziale come un'emanazione (Ausfluss) dello stesso diritto sostanziale, cioè, come la sua efficacia. A questo riguardo, distingueva il giurista tra i diritti in se stessi (Rechten an sich), i diritri vulnerati (verletzen Rechten) e i diritti in stato di difesa (im Zustand der Vertbeidigung $)^{22}$, tutti fenomeni del diritto materiale. Questo inserimento dell'azione nel piano del diritto sostanziale trascura la necessaria separazione tra questo piano e quelio del processo.

Sta di fatto che, se già non appate possibile affermare l'esistenza del proprio diritto sostanziale prima del contradittorio, a fortiori è problematica l'affermazione dell'esistenza di una 'azione di diritto sostanziale' già all' inizio della domanda. La sua esistenza potrà essere affermata solo alla fine del processo, con il transito in giudicato della sentenza. In questo momento però essa (la azione), si confonderà con la propria efficacia della sentenza.

\footnotetext{
${ }^{18}$ Pontes de Miranda, Tratado, cit, p., 67.

"Pontes de Miranda, Tratado, cit., p. 116.

2h Pontes de Miranda, Tratado, cit., P. 277.

2: Pontes de Miranda, Tratado, cit, p. 94-95, 110.

${ }^{23}$ Savigny, System des heutigen römischen Rechts, Berlin, 1841, tomo V, P. 1 e 2, 65204 e 205.
} 
Pontes de Miranda, per cercare di evitare questa contradizione, sostiene che il diritto alla pretensione di tutela giuridica (meglio: pretensione alla giustizia) non è affatto pretesa ad una sentenza favorevole ${ }^{23}$ : "Se, anzichè avere la sentenza favorevole, si ottiene quella negativa ('attore ha proposto un'azione che non gli spettava), si dichiara l'inesistenza dell'azione. Poichè l'attore non aveva l'azione, ha esercitato pretensione all rimedio giuridicoprocessuale, cioè, non poteva aspettarsi una sentenza favorevole riguardo all'azione di cui se credeva titolare" ${ }^{24}$. La spiegazione non è convincente perchè, nello stesso tempo in cui si riconosce che l'istante non aveva azione (di diritto sostanziale), si afferma che l'azione è stata esercitata tramite l' 'azione'. È palese, però, che non si può esercitare quello che non si ha.

Ovídio A. Baptista da Silva qualifica l'azione di diritto sostanziale, inerente a qualsiasi dititto, come un' "agire del titolare del diritto ai fini della sua realizzazione, prescindendo dalla volontà o collaborazione dell'obbligato" (enfasi originale) ${ }^{25}$. In altro passo, la de finisce come "l'agire di ogni dirirto, atto - indipendentemente da qualsiasi partecipazione attiva dell'obbligato - a realizzare completamente il rispettivo diritto"26. Fa notate l'autore che "la realizzazione coattiva del diritto che dispensa la volontà o collaborazione dell'obbligato è rigorosamente la stessa azione di diritto sostanziale, ossia, lo sresso agire per la realizzazione, inerente a qualsiasi diritto, con l'unica differenza che, vietata l'autotutela privata, la sua realizzazione avviene attraverso l'attuare degli organi statali. Come si vede, anzichè soppressione o sostituzione dell'azione di diritto sostanziale, si ha una vera e propria duplicazione di azioni: la prima, diretta contro l'obbligato, l'altra, verso lo Stato affinchè, una volta certificata l'esistenza del diritto, questo sia realizzato dal Potere Puhlico per mezzo della stessáa atività vietata al suo titolare privato"27.

Nonostante la generalizzazione fatta dall'autore rispetto la soppressione dell'autotutela, esistono casi in cui si esige la partecipazione attiva dell'obbligato, il che tende inveritiera o alquantomento indebolisce l'affermazione astrattamente fatta. Un'esempio ammesso, tra l'altro, da Ovídio A. Baptista da Silva, consiste nell'atto di integrare in servizio un pubblico funzionario. La sentenza non può realizzarlo direttamente ${ }^{28}$. Per restate coerente con le sue premesse, il giurista è stato costretto a negare l'esistenza della categoria delle sentenze di condanna, poichè l'agire che realizza la pretesa è l'azione di esecuzione (in caso de esecuzione dell'obbligazione) ${ }^{29}$.

23 Pontes de Miranua, Comentários ao Código de Processo Civil, t. I, Rio de Janeiro, Forense, 1974, p. XXXIV.

24 Pontes de Miranda, Comentários cit., p. XL,III-XLIV.

25 Ovidio A. Baptista da Silva, Curso de Direito Processual Civit, vol. I, cit., p. 80.

26 Ovidio A. Baptista da Silva, Curso, vol. I, 4a. ed., São Paulo, RT, 2000, p. 354-355.

${ }^{27}$ Ovidio A. Baptista da Silva, Curso, vol. If, cit., p. 84-85.

${ }^{28}$ Ovidio A. Baptista da Silva, Curso, vol. II, cit., p. 354355.

${ }^{2}$ Ovidio A. Baptista da Silva, Curso, vol. H, cit., p. 204. 
In questi casi risalta la fragilità della teoria, nella misura in cui, anche in assenza dell'azione di diritto sostanziale in queste ipotesi, la sentenza (di condanna o mandamentale) seguitebbe avendo tutta l'efficacia che le è propria. D'altta parte, non si avverte l'affermata duplicazione di azioni in alcuni giudizi costitutivi, positivi o negarivi, che sono sprovvisti di pretesa materiale, come avvienne nel caso di divorzio, annulamento di matrimonio e interdizione. In modo simile, non è pensabile aversi la duplicazione di azioni nelle azioni di mero accertamento, visto che il titolare del diritto non può agire per se stesso per la sua realizzazione - con o senza la volontà dell'obbligato la dichiarazione del proprio interessato sarebbe un flatus vocis, essendo indispensabile la certifica che avviene dall'autorità statale tramite l'esercizio della giurisdizione.

Neil'abbinare il concetto di azione di diritto sostanziale con la titolarità del diritto (un agire del titolare del diritto) già sia avverte un comprommesso con le teorie immanentiste dell'azione (Savigny) sempre rifiutato, però. Una volta messo in discussione il diritto, non si può avere a che fare con un suo titolare, ma soltanto con un sedicente avente diritto, il che può essere vero o no, a seconda della sentenza.

Oltre a ciò, l'immanenza del diritto sostanziale, che sarebbe presente in tutte le azioni di diritto sostanziale (si traterebbe della propria efficacia del diritto materiale), fa pensare alla teoria del diritto giustiziale sostanziale civile, di James Goldschmidt (anche lui un concretista). Questo diritto, composto daile norme che regolano la rutela processuale e dalle norme del diritto privato sarebbe totalmente distinto da quest'ultimo (diritto privato), poichè inteso come regolatore dall'artività del giudice (e non dci privati). Per il giurista tedesco, le norme di diritto privato si rivolgono sia ai privati che al giudice, nella misura in cui quest'ultimo deve assicurare la loro attuazione. In altre parole, il diritto privato si dirige ai privati $\mathrm{e}$ allo $\mathrm{Stato}^{3(0)}$, nel regolare la sanzione da applicare ad un trasgressore. In questa prospettiva, il diritto privato ed il diritto giustiziale sostanziale non sarebbero altro che due volti di un unico dominio giuridico ${ }^{31}$. Ancora più importante è il fatto che, così come Savigny attribuisce all'azionabilità un carattere di diritto sostanziale, anche il Goldschmidt dota l'azionabilità giustiziale civile di un significato sostanziale ${ }^{32}$. Non a caso, l'inserzione del fenomeno processuale nel diritto materiale fa diventare il concetto di azione di Goldschmidr molto simile a quello di actio del Savigny ${ }^{33}$.

\footnotetext{
${ }^{30}$ James Goldschmidt, Zivilprozessrecht, Neudruck der 2. Auflage, Berlin 1932, Aalen, Scientia Verlag, $1969, \$ 2,3$, p. 5: "Il diritto può essere visto come un'insieme di imperativi diretú à soggetti titolari di diriti oppure di regole di applicazionei dirette ai giudici. Quest'ultima caratterizzazione è adeguata al diritto giudiziale ed anche al diritto processuale civile. Da questa perospettiva, le regole giuridiche operano come notme di inibizione o di permesso di una determinata condotta dei particolati e, inoltre, cone criterio per una sentenza giudiziale di determinato contenutto."

3 James Goldschmidt, Über Begriff und Bedeutung des materiellen Ziviljustizrechts (in Zwei Beiträge zum materielfen Zivilustizrecht), in Festgabe für Heinrich Brünnex, München/Leipzig, Duncker \& Humblot, 1914, p. 109-138, esp. p. 123.

32 James Goldschmidt, Zivilprozessrecht, cit., $\$ 12,3$, p. 52: "L’azione processuale, quale oggetto concretto del processo (il metitum causae) è un diritto giudiziale di carattere materiale e non di carattere giudiziale." ${ }_{33}$ Osservazione di Andreas Kollmann, Begriffs. und Problemgeschichte des Verhältuisses von formellem und materiellem Recht, Berlin, Duricker \& Humblot, 1996, p. 598
} 
Di conseguenza, la concezione di Goldschmidt deve affrontare le stesse critiche che si indirizzano nei confronti delle idee di Savigny. Oltre a ciò, da una parte sembra essere fuori dubbio che le norme del processo civile (normalmente di diritto pubblico), sono dirette essenzialmente alla funzione giurisdizionale e non hanno a che fare con il sottostante diritto sostanziale; dall'altra parte, il soggetto della condotta prevista nella norma di diritto sostanziale non è l'organo giudiziale ${ }^{34}$.

La stessa critica può essere puntata verso l'azione di diritto sostanziale, che altro non è che una derivazione del diritto sostanziale (norme dirette al particolare) e che, nonostante ciò, dev'essere attuata nel processo tramite l'organo giudiziale, insieme con l' 'azione' processuale. L'ipotesi di una norma di condotta che contenga in se sressa il meccanismo della sua propria realizzazione giudiziale, quando trascurata, non è armonica con l'ordine giuridico brasiliano che distingue in forma netta il diritto sostanziale dal diritto processuale. Questa teoria è portata ad ammertere l'esistenza di una siffatta norma che contemporaneamente è di primo e secondo grado ${ }^{35}$.

Non fossero sufficenti queste osservazioni critiche, non si vede come possa il giudice "agire materialmente" (esercitare l'azione di diritto materiale, secondo Pontes de Miranda e Ovídio A. Baptista da Silva), in parallello con l'azione processuale: ciò che il magistrato realizza nel compiete il suo mestiere, tramite l'esercizio di competenze puhbliche attribuitagli da norme di diritto pubblico è attività assolutamente diversa da quella rerta da norme di diritto privato. Appena doppo che è stata presa la decisione è che il giudice può intervenire nel mondo sensibile. Ma a questo punto, si tratta già di efficacia della sentenza, del risulraro della tutela giurisdizionalc. Prima di ciò non si presenterebbe l'azione di diritto sostanziale. Ma, anche ques to agire del giudice non può essere equiparato all'agire del particolare, se si tiene presente la natura diversa della tutela giurisdizionale e la sua caratteristica di sostirutività, mirata a ricostruire la real tà fuori processo e non solranto a riproduria.

\footnotetext{
${ }^{34}$ Elio Fazzalari, Note in tema di Jiritto e processo, cit. p. 49, osserva contro Goldschmidt, con ragione, che "...ogni attività, quale xisulta in astratto prevista e valutata dalla noma, ha il propro soggerto, pure indicato dalla norma (...) Dalla costanza di questa indicazione astratta e tipica se trae che ograi norma riguarda una certa categoria di soggetti (quelli che vengono a trovarsi, in concreto, nelle condizioni descritre dalla norma), la quale può anche coincidere, ma non coincide setnpre e necessariamente con la generalità dei soggetti. In questi più corretti ternini, si potrà allora concludere che vi sono, nel nostro ordinamento, norme le quali indicano, como soggetto delle condotte da esse descritte e valutate, il giudice; altre norme che indicano il privato; altre norma ancora che indicano if legishatore, la pubblica amministrazione e così via. Dal che riceve, poi, conforto l'avviso, già manifestato, secondo il quale non è possibile ritenere che la norma di dirito privato s'indirizzi al privato e, al tempo stesso, al giudice che deve garantirne il funzionamento; ritenere cioè che la norma de qua regoli, al tempo stesso, la condotta del privato e la sanzione."

${ }^{35}$ Comme spiega Norberto Bobbio, Norme Primarie e Norme Secondarie, in Studi per una Teoria Generale del Diritto, Torino, Giappichelli, 1970, p.196, nella stregua di Santi Romano, i numerosi meccanismi e collegamenti di autorità e forza che produccono, modificano, applicano o garantiscono le norme giuridiche costituiscono, /sattamente, le norme di secondo grado. Ciò di cui non si era reso conto (Il Romano) era che quei "numerosi meccanismi o ingranaggi" erano costituiti anch"essi da norme, appunto dalle norme di secondo grado.
} 
6. Una volta stabilito che, seppur non si prescinde dal diritto sostanziale nello stabilire l'efficacia della sentenza, non la si può confondere col dititto di azioni sostanziale, bisogna andare oltre nell' esame del problema.

Il diritto sostanziale costituisce, certo, la materia prima con la quale opera il giudice, che, petò, in questo operare, la trasforma. La tutela giurisdizionale non presenta il diritto sostanziale in stato puro, ma qualcosa di più, di qualitativamente diverso. La sua forza ed efficacia sono altre (che quelle del diritto sostanziale non "giurisdizionalizzato") poichè si trafra di opera di ricostruzione - ed anche di creazione - e di autorità. Una conferma di ciò sj ha nel fatto di che i verbi "dichiarare", "condannare", "esecutare" e "commandare" sono tipici del diritto processuale e non hanno significa to recnico nel diritto sostanziale che, invece, parla di indenizzi, di risoluzione di contratto, tinunzia ecc. Si badi che, anche la autotutela, che sarebbe per così dire, l'azioni di diritto sostanziale in stato puro, non è equivalente alla tutela giurisdizionale quanto meno perchè, non avendo il marchio della autorità statale (eseguita dal diretto interessato, senza le garanzie del due process) può essere oggetto di futura ed eventuale revisione giudiziale.

In un siffatto contesto, l'efficacia processuale si presenta solo come una forma di tutela giurisdizionale, data a chi ha ragione, sia attore, sia convenuto (sentenza di accertamento negattivo) $)^{36}$.

D'altra parte, la distinzione tra i diversi tipi di tutela giurisdizionale non è arbirraria.

Si deve osservare, soprattutto, i principi della effettività e della sicurezza ${ }^{37}$ (quest'ultimo derivaro dal proprio concetto di Stato di Diritto e materializzato dalla nozione di due process of law), entrambi di stampo costituzionale, nonchè la situazione sosranziale dedotta in giudizio. Sono questi principi, insieme alle specificità di ciascun ordinamento giuridico, che determinano, i possibili tipi di tutela giurisdizionale in abstracto.

${ }^{36} \mathrm{Da}$ un'altra perospettiva, osserva giustamente Flávio Luiz Yarshell Tutela jurisidicional específica nas obrigaçōes e declaraçōes de vontade, São Paulo, Malheiros, 1993, p. 19 che, nonostante si pensi alla tutela giuridica come protezione, essa esiste anche rispetto a quello che è stato vinto. E questo perchè lo Stato glile ha assicurato, tramite l'esercizio delia funzione giurisdizionale e del due process of law, la soluzione del conflitto in base a regole precstabilite e che garantiscono la sua partecipazione (contradittorio) tendente al risultato finale, che esiste, esattamente per impedire la supremazia del più forte su il più debole.

${ }^{37}$ Nonostante sia diventato un luogo comune, la considerazione di Chiovenda, Della azione nascente dal contratto preliminare, in Saggi di diritto processuale civile, Roma, Foro Italiano, 1930, vol. 1, p. 110, mantiene tutta la sua attualità: "il processo deve dare per quanto è possibile praticantente a chi ha un dirito tutto quello e proprio quello ch'egli ha dixitto di conseguire." . Luiz Guilherme Marinoni, Tutela Inibitória (individual e coletiva), 3a. ed., São Paulo, RT, 2003, p. 449, benchè presentando una posizione diversa, pone in rilievo nel il fatto che la tutela giurisdizionale dev'essere classificata a partire dai quello che effettivamente fa per realizzare il diritto materiale, e non appena solo in base al tipo di effetto giuridico dichiarato. Osserva che la preocupazione dev'essere quella di analizzare il risultato che il processo deve presentare. Sull'effettività e la sua base costituzionale, si vedano le osservazioni di Marcelo Lima Guerra, Execução Irydireta, São Paulo, RT, 1998, P. 48-54. 
In concreto, la tutela dipende dalla tichiesta fatta dall'attore e dalle eccezioni di diritto sostanziale avanzate dal convenuto. A causa del principio del dispositivo, diventa possibile una pluralità di sentenze e di richieste per un singolo rapporto di diritto sostanziale. Si prenda come esempio la possibilità di chiedete soltanto l'accertamento della situazione di diritto sostanziale, laddove è già avenuta la violazione del diritto (ex vi art. $4^{\circ}$, par. unico del CPC brasiliano). La violazione del diritto potrebbe dare causa anche ad una domanda mandamentale, di condanna od esecutiva. Ma non per questo si può affermare che la classificazione delle sentenze sia una questione puramente processuale ${ }^{38}$.

D'altra parte, si deve osservare che il giudice brasiliano è autorizzato, a regola dell'art. $461 \mathrm{del} \mathrm{CPC}$, a non attenersi strettamente a quanto richiesto. Se, la tutela specifica dell'obbligazione (normalmente di carattere ordinatorio) non è giunta ad un risultato soddisfacente, il magistrato può prendere delle misure che conducano a risultati equivalenti, anche attravetso provvedimenti di natura esecuttiva ${ }^{39}$.

Si deve, tenendo presente quanto detto, preferire la tutela che garantisca in più alto gxado l'efettività. Dal punto di vista della sicurezza, importa essenzialmente che il convenuto possa esercitare in modo adeguato il suo diritto di diffesa. La situazione giuridica sostanziale non può impedite l'eficacia che si ptetende. Si prenda come esempio il problema delle obbligazioni pecuniarie. In tale ipotesi, la turela rappresentata da una sentenza di condanna è la più adatta, poichè la futura esecuzione surrogattoria a che da causa, è normalmente la più effetiva.

L'ambito più adatto alla utilizzazione della tutela mandamentale è quello dei dititti assoluti, nella misura in cui essi danno origine al dovere negativo di astensione dall'invadete Ia sfera giuridica altrui, alterum non laedere $e^{40}$.

Non esiste, però, mottivo per determinate un vincolo necessario tra diritti assoluti c sentenze mandamentale ${ }^{41}$. Oltre essi, le obbligazioni di fare e di non fare possono essere oggetto di questo tipo di provvedimento giurisdizionale. Anche in ordine ai diritti relativi si riconosce odiernamente il dovere di astenzione: ogni diritto è relativo in rapporto al suo oggetto e assoluto per quanto tiguarda la sua esigenza di inviolabilità da parte di qualsiasi

\footnotetext{
${ }^{38}$ Come sostengono, v.g, Rosenberg/Schwab, Zivilprozessrecht, 12a. ed., München, Beck, 1977, \$ 93, 3, p 478. 39 Interessante, a questo riguardo, l'esempio di Cândido Rangel Dinamarco, A Reforma da Reforma, 4a. ed., São Paulo, Malheiros, 2002, p. 228: "Il giudice condanna (rectius: commanda ordina, nella proerspettiva qui presentata) un negozio notturno a ridurre fino a livelli sopportabili i rumori che produce. Diventa res iudicata e il convenuto non la osserva." fl giudice "può arrivare al punto di ordinare la chiusura dello stabilimento", anche senza richiesta dell'attore.

4) Luiz Guiherme Marinoni, Tutela Inibitória, cit, p. 415.

"Luiz Guilherme Marinoni, Tutela Inubitória, cit, p. 418. Eduardo Talamini, Tutela Relativa aos Deveres de Fazer e de Não Fazer, São Paulo, RT, 2001, p. 125-127 relativizza la distinzione tra diritti e doveri e sostiene, giustanente, lapplicazione dell'art. 461 del CPC sia ai doveri che alle obbligazioni di fare e non fare.
} 
soggetto La distinzione ha a che fare soltanto con la forma tramite la quale si realizzano indipendentemente dalla collaborazione altrui. I diritti relattivi, nci rapporti con gli altri il cui comportamento è strumentale per la sua realizzazione $e^{42}$. La spiegazione è giusta in parte, perchè nelle obbligazioni di fare non si sta davanti a un dovere di astensione. In questa sede, la tutela mandamentale trova la sua giustificazione nel principio della effettività.

Comunque sia, la questione fu grandemente facilirata nel diritto brasiliano a causa della nuova disciplina data al compimento delle obbligazioni di fare o di non fare, apportata dal nuovo art. 461 del CPC, in particolare, dal suo \$1o (Legge n. 8.952, del 13.12.1994). Con essa è stato capovolto il principio neminem praecise potest cogi ad factum: "L'obbligazione si converte in pagamento a richiesta dell'auttore o se impossibile la turela specifica o l'ottenimento del risultato prattico corispettivo." Si evitò, così, l'equivoca esegesi dei glossarori, privilegiandosi la forza del negozio giuridico o del contratto, fino al punto, ovviamente, in che questa realizzazione è compatibile con il rispetto della dignità del debitore.

Se l'obbligazione di fare è personalissima, o fu convenuto che l'adempimento non sarebbe stato specifico, si mostra adeguata la tutela di condanna. Nell'obbligazione di fare fungibile, che per ipotesi può essere realizzata da un terzo a carico dell'obbligato, l'autore può scegliere tra la sentenza di condanna e quella mandamentale. È questo il significato dalla multa, stabilita nell'articolo $287 \mathrm{del}$ CPC (con redazione secondo la legge n. 10.444, del $07.05 .2002)^{43}$.

La tutela di accertamento ha che fare solo con la verifica dell'esistenza o inesistenza di un rapporto giuridico (eccezionalmente si può avere dichiarazione sulla falsità di un documento). L'accertamento di un mero fatto o dell'esegesi di una norma in astratto considerata (la cui fattispecie non si ha verificato) comprometerebbe la garanzia di difesa del convenuto, sia perchè un singolo fatto può servire ad diversi effetti giuridici, sia perchè la norma generale ed astratta può essere applicała ad una serie indefinita di situazioni concrete ${ }^{44}$.

La tutela esecuttiva lato sensu ha che fare soltanto con il passare dalla sfera giuridica del debittore a quella del creditore ciò che in essa dovrebbe essere già - linea di divisione della tutela esecuttiva lato sensu e quella di condanna, secondo Pontes de Miranda. La tealizzazione della tutela di condanna, però, perchè si dirige genericamente al patrimonio del debittore, esige più ampie possibilità di difesa (principio della sicurezza) e, di conseguenza, un processo autonomo di esecuzione ${ }^{45}$.

\footnotetext{
42. Marco Comporti, Diritu reali in generale, p. 26-27, apud Marinoni, Tutela Inibitória, cit., p. 417.

${ }^{43}$ Marinoni, Tutela Inibitória, cit., p. 460 462, i base specialmente al principio della effettività

${ }^{44}$ Andrea Proto Pisani, Appunti sulla Giustizia Civile, Bari, Cacucci, 1982, p. 93. "uno stesso fatto può essere rilevante ai frni di più e diversissimi effetti giuridici (...) una tale norma per definizione è suscettibile di trovare applicazione com riferimento ad uma serie pressoché indefinita di fattispecie concrete."

${ }^{45}$ L'introduzione dell'articolo $461-A$ e la nuova redazione dell'articolo 621 del $C P C$ brasiliano (Legge 10.444 , del 7.5.2002), riconoscono, de lege lata, e in modo generico, la tutela esecuttiva lato sensu. Con ciò si prestigia in modo deciso il valore efetività, ma non a scapito del diritto di diffesa del convenuto, tra l’altro riconosciuto costituzionalmente.
} 
La tutela mandamentale, nonostante operi a somiglianza di quella esecutiva lato sensu (tramite l'emissione dì ordini del giudice), è alquanto diversa da essa perchè agisce sulla volontà dalla parte e non sul suo patrimonio. E questo è esigenza della situazione sostanziale che non racommanda (se si parte dalla perspettiva della più gtande efettività) l'uso della condanna ${ }^{46}$.

Infatti, sia la tutela esecutiva lato sensu, sia quella nandamentale, sono dirette al principio della maggiore effettività possihile. Basta osservare, quanto a quest'ultima, che non sarebbe possibile ottenersi il risultato specifico per nezzo della semplice condanna, poichè essa condurrebbe soltanto alla ottenzione dell'equivalente pecuniario (uso dei mezzi di esecuzione surrogattori).

D'altra parte non sembra giusto confonderle con la tutela di condanna, supostamente perchè anch'essa conterebbe un'ordine di prestazione, cambiando, in questo caso, soltanto la forma di realizzazion $e^{47}$. Il fatto è che in questa ipòtesi la sentenza non contiene in sè ordine di qualsiasi natura, ma solo un giudizio di rimptovero. Si tratta soltanto di un'esortazione al convenuto, che non da margine a qualsiasi sanzione, penale o civile.

Si sostiene, inoltre, che la sentenza mandamentale costituisce titolo per l'esecuzione forzara, così come quella di condanna. Sarebbe perciò una condanna di particolare tipo, con un contenuto diverso solo in quanto la sanzione in essa innestata mette in evidenza l'elemento di comando. A ben guardare, però, la differenza sta nel contenuto della sentenza, giacchè i due verbi "comandare" e "condannare" hanno significato e conseguenze giuridiche diverse: it comando (1'ordine) agisce sulla volontà del convenuto, mentre la condanna sul suo patrimonio in un processo futuro (effetto esecuttivo della sentenza), tramite mezzi surrogatori. Nel caso del comando, non si ha propriamente esecuzione nella misura in cui la soddisfazione del creditore è ottenuta per mezzo della collaborazione del convenuto, costretto ad eseguire la sua obbligazione per evitare mali maggiori ${ }^{48}$.

Si deve inoltre sottolineare che tra i cinque tipi di tutela (accertamento, condanna, costituzione, ordine e esecuzione lato sensu), solo le sentenze di accertamento e costiruttiva soddisfano in sè stesse la pretesa processuale, senza bisogno di qualsiasi atto ulteriore; la sentenza di condanna resta a metà strada e crea soltanto le condizioni giuridicbe (titolo esecutivo) perchè questa soddisfazione avvenga in processo autonomo ed independente, detto di esecuzione. Infine, le sentenze mandamentale e di esecuzione soddisfano la pretesa nello stesso processo, tramite atti materiali che producono effetti nel mondo fattuale

Infine, nel caso di tutela mandamentale o esecuttiva lato sensu, l'attività giurisdizionale si conclude soltanto con ia realizzazione del diritto affermatosi nella sentenza. L'articolo 463 del CPC brasiliano impedisce solo l'alterazione della sentenza dopo la sua pubblicazione.

\footnotetext{
"In modo diverso, Marinoni, Tutela Inibitória, cit, p. 398. Pex questo autore, il criterio per definire la qualità mandamentale di una sentenza è puramente processuale.

${ }^{47}$ Questa é la tese di Humberto Theodoro Júnior, Curso de Direito Processual Civil, vol. \}, cit., $\mathrm{n}^{0} 497$, p. $468-469, n^{\circ} 499$, p. 470 , già presentata supra.

${ }^{48}$ Liebman, Processo de Execução, 3a. ed., São Paulo, Sałaiva, n. 3, p. 6.
} 